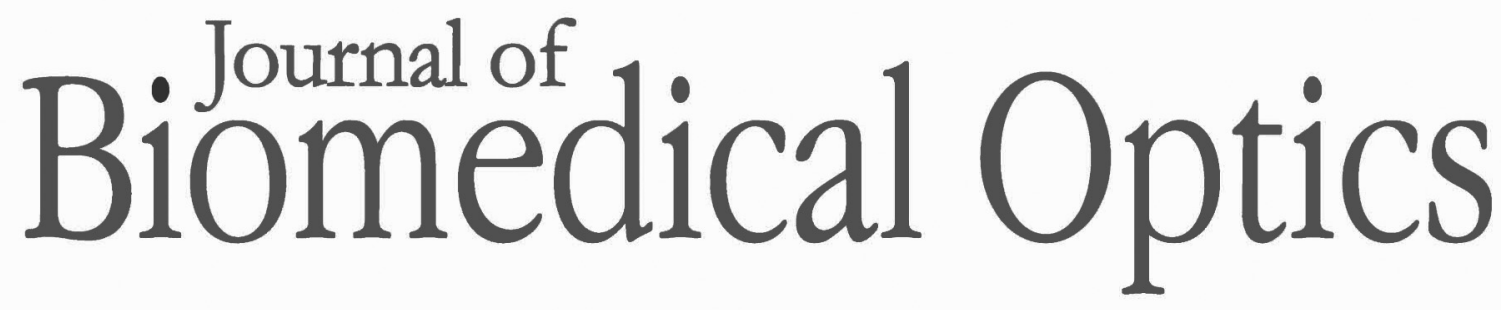

\title{
Optodynamic monitoring of laser tattoo removal
}

Boris Cencič

Ladislav Grad

Janez Možina

Matija Jezeršek 


\title{
Optodynamic monitoring of laser tattoo removal
}

\author{
Boris Cencič, ${ }^{b}$ Ladislav Grad, ${ }^{b}$ Janez Možina, ${ }^{a}$ and Matija Jezeršek ${ }^{a}$ \\ aUniversity of Ljubljana, Faculty of Mechanical Engineering, Ljubljana, Slovenia \\ ${ }^{\text {b} F o t o n a ~ d . d ., ~ L j u b l j a n a, ~ S l o v e n i a ~}$
}

\begin{abstract}
The goal of this research is to use the information contained in the mechanisms occurring during the laser tattoo removal process. We simultaneously employed a laser-beam deflection probe (LBDP) to measure the shock wave and a camera to detect the plasma radiation, both originating from a high-intensity laser-pulse interaction with a tattoo. The experiments were performed in vitro (skin phantoms), ex vivo (marking tattoos on pig skin), and in vivo (professional and amateur decorative tattoos). The LBDP signal includes the information about the energy released during the interaction and indicates textural changes in the skin, which are specific for different skin and tattoo conditions. Using both sensors, we evaluated a measurement of threshold for skin damage and studied the effect of multiple pulses. In vivo results show that a prepulse reduces the interaction strength and that a single strong pulse produces better removal results. @ 2012 Society of Photo-Optical Instrumentation Engineers (SPIE). [DOI: 10.1117/1.JBO.17.4.047003]
\end{abstract}

Keywords: laser; tattoo removal; photothermolysis; monitoring.

Paper 11654P received Nov. 7, 2011; revised manuscript received Jan. 17, 2012; accepted for publication Feb. 16, 2012; published online Apr. 11, 2012.

\section{Introduction}

Removal of intradermal tattoos embedded in human skin has become successful when $Q$-switched lasers in the visible and near IR were introduced as the primary modality. The basic principle of laser tattoo removal is selective photothermolysis where selective absorption of laser light by the tattoo pigment is achieved, and the pulse duration is less than the thermal relaxation time of the target. ${ }^{1}$ Commonly used lasers for tattoo removal are the $Q$-switched ruby $(694 \mathrm{~nm})$, alexandrite $(755 \mathrm{~nm})$, and $\mathrm{Nd}$ : YAG (1064 and $532 \mathrm{~nm}$ ) lasers with typical pulse duration from 5 to $100 \mathrm{~ns}$ and with fluences from 1 to $12 \mathrm{~J} / \mathrm{cm}^{2}$ (Refs. 2 and 3). However, the therapy still involves multiple treatment sessions and occasional complications such as dyspigmentation, allergic reactions, ink darkening, epidermal debris, and potential carcinogenicity. ${ }^{2-4}$ Therefore, there is constant research into the physical mechanisms of the laser-tattoo-tissue interactions, which are not well understood and remain to be evaluated. ${ }^{2,3}$

Since the tattoo pigment is highly absorptive for the applied laser wavelength, ${ }^{5}$ plasma formation takes place at laser fluences mentioned above. Darwin et al. ${ }^{6}$ used a computer simulation to study the laser-pigment interaction. They found that the tattoo pigment disintegration is driven by strong acoustic waves inside the particles. Since shorter laser pulses are more efficient, there is expectation regarding picosecond lasers, which promise better tattoo removal rates. ${ }^{7,8}$ Cavitation bubbles, which can damage the surrounding tissue, are formed around the pigment particles due to their increased temperature and plasma formation. This is in general agreement with histologic and electron microscopic analyses of biopsies. ${ }^{7,9-12}$ The pigment breakup is only one stage in its successful removal. Several mechanisms are assumed possible. The smaller fragments may be phagocytosed and subsequently eliminated by the lymphatic system. ${ }^{11}$ Another possible removal process is pigment chemical decomposition

Address all correspondence to: Matija Jezeršek, University of Ljubljana, Faculty of Mechanical Engineering, Ljubljana, Slovenia. Tel: 0038614771171 ; Fax: 003861 2518 567; E-mail: matija.jezersek@fs.uni-lj.si into gaseous products. ${ }^{6}$ Direct ablation of the pigment can also take place, with skin damage as a negative side effect. Physical alteration and redistribution of the pigment may also account for its reduced visibility. ${ }^{7,12}$

In order to set optimal parameters for the laser tattoo removal, it is important to know the properties of the tissue and the pigment. Optical properties and the pigment depth distribution are among the most important factors. Various noninvasive measurement techniques are used to evaluate those properties. These include optical coherent tomography, ${ }^{13}$ confocal scanning laser microscopy, ${ }^{10}$ and pulsed photothermal radiometry. ${ }^{14}$ All of these techniques can analyze the tissue composition before and after laser irradiation. As it is frequently necessary to deal with huge differences within the same tattoos (a type of tattoo pigment, depth distribution, skin type, etc.), it is appropriate to apply a method that monitors the interaction during a single laser pulse. Although many methods are used in investigations of pulsed laser ablation, such as fast brightfield and Schlieren photography ${ }^{15}$ and laser-beam deflection probe, ${ }^{16-18}$ we were not able to find their use for the purpose of real-time monitoring of the laser tattoo removal process in the literature.

By considering the laser tattoo removal as an optodynamic process, ${ }^{19}$ we developed a novel methodology to monitor tattoo ablation using two measuring techniques: a fast laser beam deflection probe (LBDP), which measures the shock or optodynamic wave and a near infrared (NIR) camera, which visualizes the plasma radiation, both generated by high-intensity pulsedlaser illumination of the tattoo. The experiments were performed in vitro (skin phantoms), ex vivo (marking tattoos on pig skin), and in vivo (professional and amateur decorative tattoos on several patients).

\section{Experimental Setup}

The experimental setup is presented in Fig. 1. We used a $Q$-switched Nd:YAG laser delivering 6 ns FWHM pulses

0091-3286/2012/\$25.00 (C) 2012 SPIE 
with a top hat profile through a seven-mirror articulated arm. ${ }^{20}$ Only the 1064-nm wavelength, which has high absorption in black or dark blue tattoos, was used. The end of the arm was fixed to the measuring setup to obtain repeatable measurement conditions. The laser beam diameter was $4 \mathrm{~mm}$ in all experiments.

The laser tattoo removal process was monitored using two techniques: The optodynamic waves in the surrounding air were measured by the laser-beam deflection probe (LBDP); the plasma radiation, emitted from the irradiated area, was detected by the NIR camera with a visible and near-infrared spectral sensitivity. Additionally, the long-term effects of the laser removal process were analyzed after six weeks by digital photography. Each technique is described in detail below.

\subsection{Optodynamic Wave Measurement by the Beam Deflection Probe}

During the laser-pulse tissue irradiation, significant thermal and mechanical transients are generated when high-fluence nanosecond pulses are used. Consequently, shock waves are evolved during the expansion of the plume into the surrounding air. ${ }^{21}$ These waves were detected by the deflection of a HeNe laser $(\lambda=633 \mathrm{~nm})$ beam. The laser beam (diameter $0.8 \mathrm{~mm})$ passes the treated surface at a distance of approximately $6 \mathrm{~mm}$. To achieve better spatial resolution, an achromatic lens (Edmund Optics, $f=40 \mathrm{~mm}$ ) is used to focus the probe beam to a radius of $25 \mu \mathrm{m}$. The beam waist position is aligned with the Nd:YAG laser beam. The beam deflection was measured by a bipolar photodiode with a cutoff frequency of $100 \mathrm{MHz}$, connected to a digital $600 \mathrm{MHz}$ oscilloscope.

The LBDP technique provides quantitative information about the average speed of the optodynamic wave and its amplitude. Both are correlated with the energy released during interaction. ${ }^{16}$ A typical optodynamic waveform measured by the LBDP is shown in Fig. 2, with plain white paper as the target surface. The peak represents the positive pressure gradient disturbance. The negative part of the signal is much longer and represents the negative pressure gradient disturbance, which

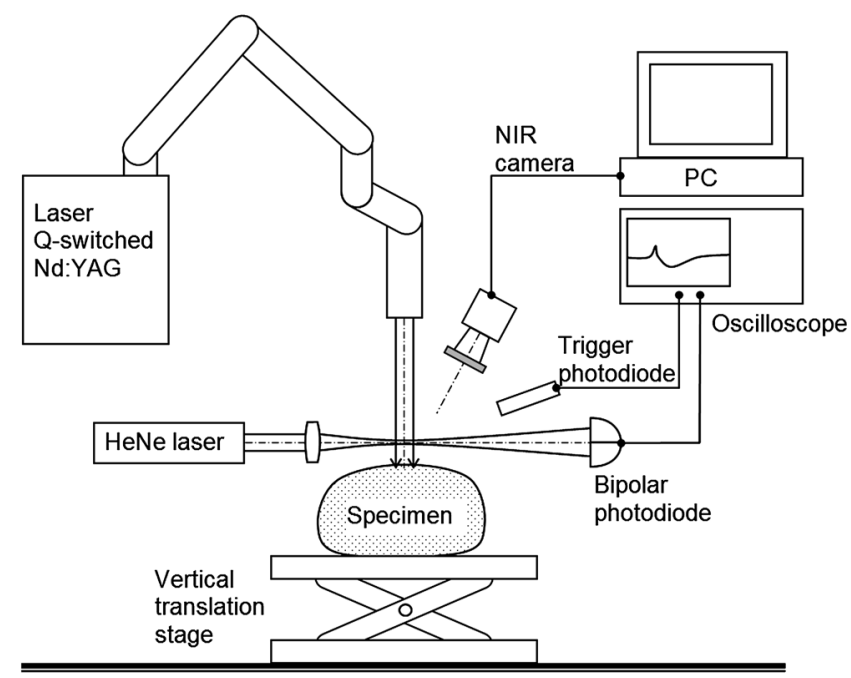

Fig. 1 The experimental setup for monitoring the laser tattoo removal process.

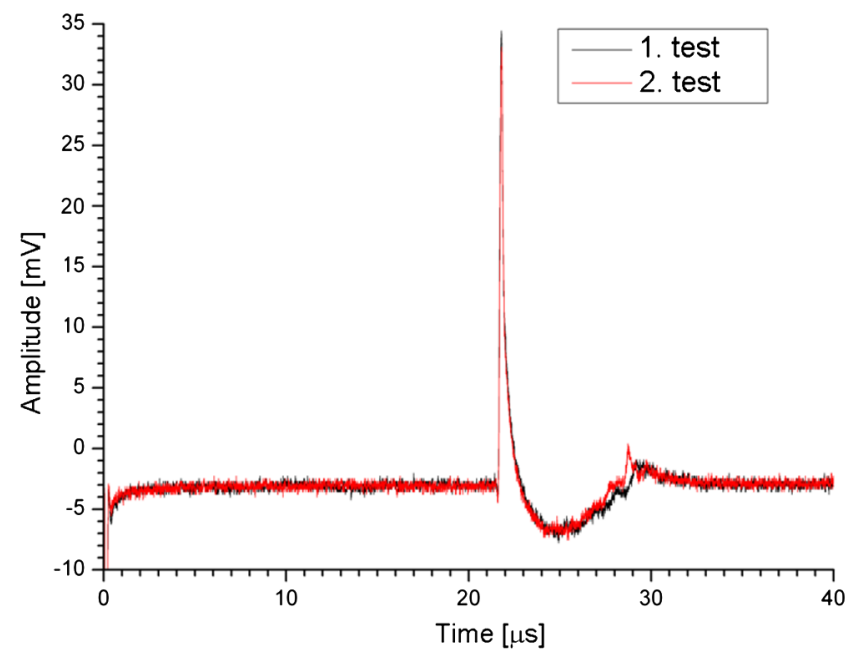

Fig. 2 Typical optodynamic waveform measured by the laser beam deflection probe. Distance between target surface and probe-beam was $8 \mathrm{~mm}$.

follows after the shock front. The peak is quite sharp, indicating a fast response of the LBDP sensor.

\subsection{Plasma Measurement With The Nir Camera}

In the case of laser tattoo removal, the volumetric energy density can be high enough to initiate plasma formation. We used a NIR camera for measurement of this plasma radiation. The camera (PointGrey, FireFly MV, $640 \times 480$ pix, $60 \mathrm{fps}$ ) was triggered at the same time as the laser flash lamp, and the shutter time was adjusted to $10 \mathrm{~ms}$ so that the emitted light during the entire process (illumination and setting down) was acquired. The camera was equipped with a lowpass optical filter (KG5 filter glass) with sufficient optical density to completely block out the laser light. Figure 3 shows typical images of plasma radiation, acquired by the NIR camera.

A time-integrated signal of plasma radiation (Isum) was calculated from the acquired images. The values are calculated as a sum of pixel values over the entire image. The sum was used to estimate the interaction strength of the laser light with the absorbing medium. The spatial distribution of the measured intensity was used to study how uniform the plasma formation was over the relatively large irradiated surface.

The NIR camera was also used to determine the exact distance between the skin and the LBDP by the triangulation principle. ${ }^{22}$ This distance $\left(h_{m}\right)$ was further used in correcting the arrival time of the shock wave according to the nominal distance of the LBDP beam from the interaction site $\left(h_{m}\right)$ :

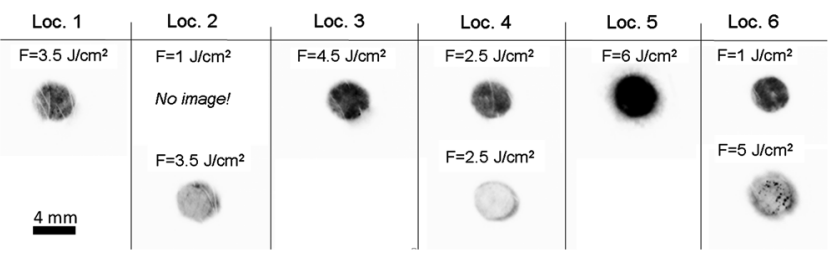

Fig. 3 Examples of plasma radiation detected during in vivo tattoo removal. Images in the second row represent the effect of the second laser pulse on the same area. All images have inverted grayscale. 


$$
\Delta t=\frac{h_{n}-h_{m}}{c_{\text {sound }}} .
$$

We assumed that the shock-wave speed at nominal distance was reduced to the speed of sound $\left(c_{\text {sound }}=340 \mathrm{~m} / \mathrm{s}\right)$. The LBDP signals, presented in Fig. 4, are corrected along time axis using this correction.

\subsection{Tattoo Removal Rate Measurement}

The tattoo removal rate was measured in vivo as a difference between the final (after six weeks) and the initial tattooed skin lightness by using a commercial camera (Nikon D90). The lighting conditions during the image acquisition of tattoos were controlled by assuring that only indoor light (fluorescent lamps) was present. Also, the same room and the same camera settings were used. During the image analysis, the RGB color was transformed into a HSV color space (see Fig. 5), where we take the $V$ component (value or lightness) into further calculation. We define the contrast of the observed area as:

$$
C_{i}=\frac{V_{\text {skin }}-V_{i}}{V_{\text {skin }}-V_{\text {black }}}
$$

where index $i$ indicates the illuminated tattoo area number, $V_{i}$ stands for the average lightness of the observed area, $V_{\text {black }}$ stands for the average lightness of the darkest tattoo

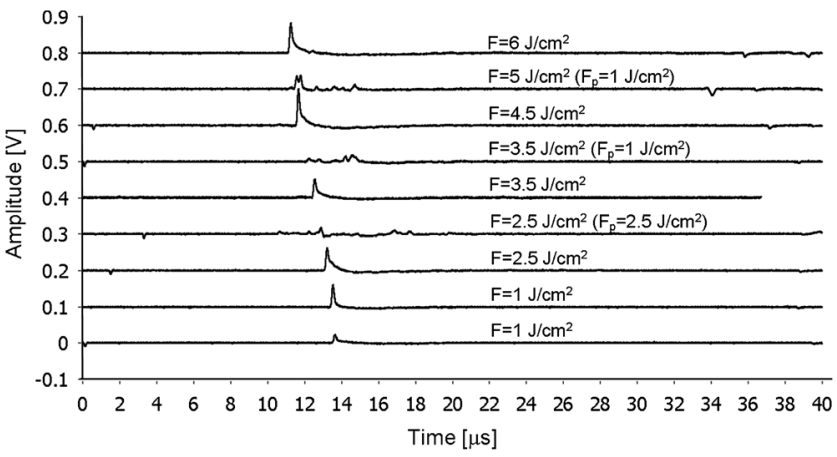

Fig. 4 The LBDP signals measured in vivo at different fluences. area on the photo, and $V_{\text {skin }}$ stands for the average lightness of the surrounding skin. The average lightness of the area $\left(V_{i}\right)$ was measured as the average pixel value within a rectangular region $(2 \times 2 \mathrm{~mm})$ positioned inside the illuminated area. The same procedure was used to measure the $V_{\text {skin }}$ and $V_{\text {black }}$, where the averaging region did not interfere with the tattoo-skin boundary. Defined this way, the tattoo is completely removed when it has zero contrast, but the change of contrast cannot exceed the initial contrast. The difference between the final and the initial tattoo contrast is therefore:

$$
\Delta C_{i}=-\left(C_{i . \text { after }}-C_{i . \text { before }}\right),
$$

where the same areas are taken into consideration before and after the removal procedure.

\section{Experiments}

The experiments were performed ex vivo, in vitro, and in vivo. Tattooed marks on pig skin were used for the ex vivo measurements. In such experiments pig skin is assumed to be a good replacement for human skin. ${ }^{21}$ The advantage of using these samples was their availability and the possibility to use laser parameters beyond their safe range. Collagen skin phantoms were used for the in vitro measurements. The samples were prepared as described in Ref. 14. Collagen gel was prepared by dissolving $1.25 \mathrm{~g}$ collagen powder (bovine skin, Sigma-Aldrich) in $6 \mathrm{ml}$ of water with $0.2 \%$ of formaldehyde, which increases the mechanical strength at room temperatures. The $200-\mu$ m thick pigment layer was positioned between two 1-mm-thick layers, composed of transparent collagen. The middle absorbing layer was prepared as neighboring layers with the addition of 2-ml black India ink as the absorber. No attempt was made to simulate either the scattering of the skin or the depth distribution of the pigment. While the skin phantom is very different from real skin, the expected advantage was a clearer view due to the absence of scatter, uniformity of the pigment distribution, and repeatable results on different positions on the sample.

The measurements in vivo were performed on the tattooed skin on the right forearm of one patient with no previous removal attempts. The tattoo was black and amateur in origin. We selected 15 locations with homogeneous and comparable tattoo lightness. The average value $C_{i \text {.before }}$ of all locations
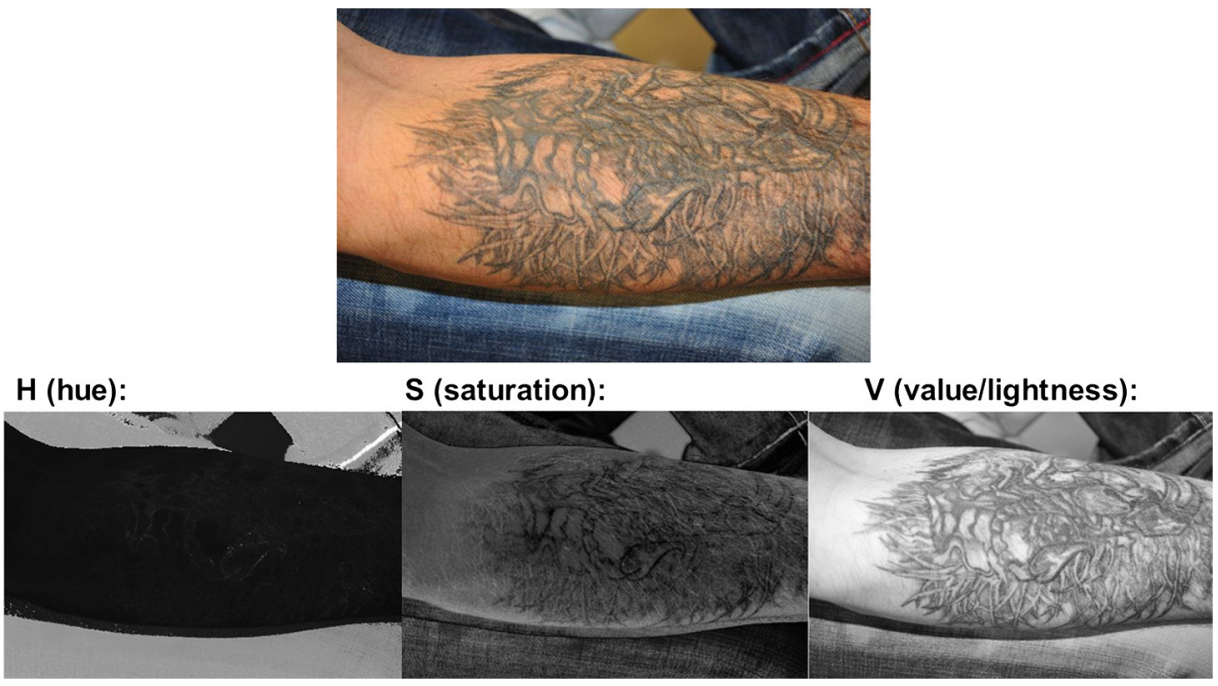

Fig. 5 Photo of the tattooed forearm in RGB (upper image) and a separate presentation of $H, S$, and $V$ channels. 
was $75 \%$, and the standard deviation was $10 \%$. The forearm was held in place at the position where the laser beam and the sensors were pointing. Before the laser procedure, a color image of the tattooed area was taken. During the procedure the initial status of the treated area was first recorded with the NIR camera. Signals from the NIR camera and from the LBDP were recorded at the time as the tattoo-removing laser pulse was applied. The immediate whitening of the skin was recorded with the NIR camera 5 to $10 \mathrm{sec}$ after the laser pulse, and after 10 to $15 \mathrm{~min}$ a color image was also taken. To assess the longterm removal effect, another color image was taken after six weeks.

For the ex vivo and in vitro measurements, the initial situation was recorded by taking a color image of the tattooed spots that were to be treated. During the laser procedure, the LBDP and the NIR camera signals were recorded. Another image of the illuminated area was taken after 10 to $15 \mathrm{~min}$.

With all samples we observed the ablation effects in the skin as a function of laser fluence and the effect of multiple pulses on the same irradiated spot with the time interval of 10 to $15 \mathrm{sec}$.

\section{Results and Discussion}

For the in vivo experiments we used the range of fluences, which were considered safe based on previous experience, from approximately 1 to $6 \mathrm{~J} / \mathrm{cm}^{2}$. The effect of multiple pulses was studied by applying two pulses with the total fluence comparable to a single pulse. Whitening of the treated skin area was observed immediately after the laser pulse illumination in all cases. This effect is caused by rapid local heating of the pigment leading to plasma and gas formation and subsequent dermal and epidermal vacuolization. ${ }^{2,6}$ At the highest fluence $\left(6 \mathrm{~J} / \mathrm{cm}^{2}\right)$ a minor bleeding was observed in one case. Otherwise, there was no long-term scarring, textural changes, or hypopigmentation. Figure 3 shows typical images of plasma radiation for different tattoo areas, acquired by the NIR camera. We can see uneven light intensity distribution, which reflects the uneven pigment distribution and also the presence of laser speckles, which localize plasma formation. ${ }^{21}$ There was also a considerable amount of light emitted from the neighboring area of the illuminated spot, which is a consequence of tissue scattering properties. This is especially evident on images of second pulses, where the plasma diameter is approximately $10 \%$ bigger than at first pulses. This shows that the tissue has increased scattering and reduced absorption after the first laser pulse. The diagram in Fig. 6 shows the relation between the time-integrated plasma radiation over the entire image (Isum) and the laser

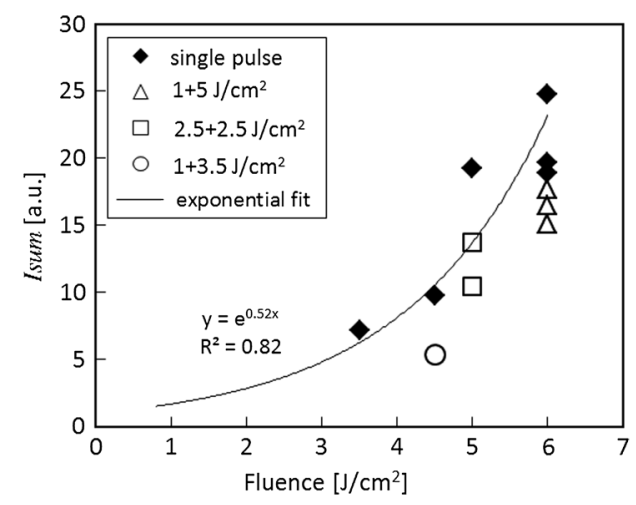

Fig. 6 The time-integrated plasma radiation (Isum) as a function of laserpulse fluence. pulse fluence. It is evident that the plasma emits more light at higher fluences and in the case of the first laser pulse. Exponential curve, fitted on single-pulse data points, is added to show the correlation.

Optodynamic signals, measured with the LBDP, have shorter peak arrival times for higher fluences (Fig. 4). The peak amplitude is roughly proportional to the applied fluence in the case of a single pulse. In the case of double pulses, the second pulse produces a smaller and more complex signal. For the double pulses only the signals resulting from the second pulse are shown on the graphs, with the fluence of the first pulse in parentheses.

Compared with a single pulse of the same cumulative fluence, the response to the main pulse of the two-pulse sequence was much weaker both on the NIR camera shot and on the LBDP signal. The LBDP signal shows multiple peaks, which we attribute to structural changes in the skin, produced by the first pulse.

We can also see smaller peaks at time greater than $34 \mu \mathrm{s}$ obtained at fluences above $4.5 \mathrm{~J} / \mathrm{cm}^{2}$. We attribute these peaks to ejected tissue particles, which intersect the LBDP probe beam. The signal resulting from the optodynamic wave is produced by the pressure and the resulting refractive index gradient, while the signal resulting from particle ejection is a consequence of the direct blocking of the probe beam. The delay of the signal attributed to particle ejection is consistent with the fact that particles move much slower than the optodynamic wave. The actual value of the fluence at which this happens also depends on the darkness of the tattoo. At the fluences used in vivo we did not notice any indication of skin perforation.

An important advantage of the in vivo measurements was that the possibility to assess the long-term removal effect by measuring the difference between the final and the initial tattoo contrast (see Sec. 2.3). It turned out that the long-term effect was better when the laser energy was administered to a particular spot in a single pulse. This correlation is shown on Fig. 7, where the exponential curve is fitted using single-pulse data points. The best removal rate was obtained with the highest applied fluence of $6 \mathrm{~J} / \mathrm{cm}^{2}$. The same cumulative fluence, delivered in a prepulse and a main pulse with the fluence of 1 and $5 \mathrm{~J} / \mathrm{cm}^{2}$, for example, had a smaller effect. Exceptions are two data points of $2.5+2.5 \mathrm{~J} / \mathrm{cm}^{2}$ double pulses, which are located near the expected position or even higher. The $1+3.5 \mathrm{~J} / \mathrm{cm}^{2}$ double pulse has nearly the same effect as a single $4.5 \mathrm{~J} / \mathrm{cm}^{2}$ pulse, but the tattoo bleaching is nearly zero.

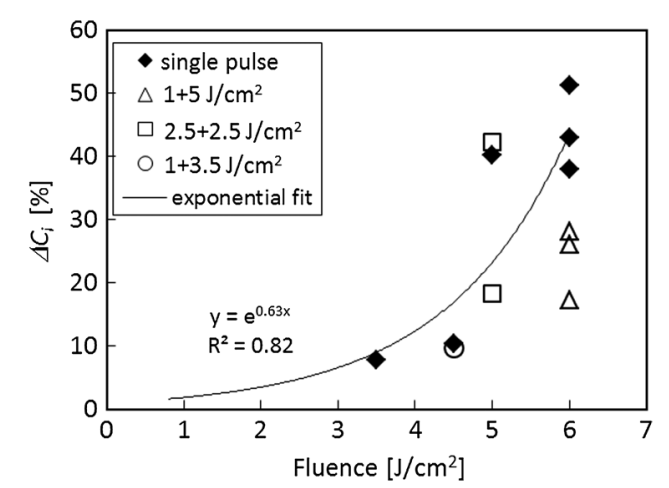

Fig. 7 Tattoo bleaching at different fluences. 
Figure 8 presents the relation between the plasma radiation and the long-term tattoo bleaching, which is a significant result. The solid rhomboid points are the data, obtained at locations with single-pulse illumination and the points with no fill are the data of double-pulse illumination. In the latter case, the plasma radiation (Isum) is the sum of both pulses. The linear fit is calculated over single-pulse-points and shows high correlation $\left(R^{2}=0.82\right)$. That means that the long-term tattoo removal rate can be estimated by measuring the time integral of plasma irradiation (Isum). According to these results we assume that each laser pulse that generates plasma also removes some tattoo pigment, being the first or the following one. Of course, other effects related to the skin damage must be controlled, and further experiments need to be done to prove these preliminary conclusions.

Ex vivo we measured the response of the untreated and tattooed pig skin to laser pulses of different fluences. We studied the response to single-pulse illumination where the fluence was varied from 1 to $8 \mathrm{~J} / \mathrm{cm}^{2}$. The untattooed skin yields a weak but measurable signal up to $6 \mathrm{~J} / \mathrm{cm}^{2}$ and a distinct threshold at $7 \mathrm{~J} / \mathrm{cm}^{2}$ (Fig. 9). The signal above the threshold shows a typical optodynamic response. We assumed that this undesired interaction also determines the damage threshold, although no visible damage to the skin occurred. We can also see that the LBDP response for fluences from 1 to $6 \mathrm{~J} / \mathrm{cm}^{2}$ has little statistical variation, which indicates that the untattooed skin has uniform properties independent of the location on the sample.

The tattooed pig skin shows a markedly different response at much lower fluences (Fig. 10). The signal at the fluence of $2 \mathrm{~J} / \mathrm{cm}^{2}$ is larger and of a more complex shape compared

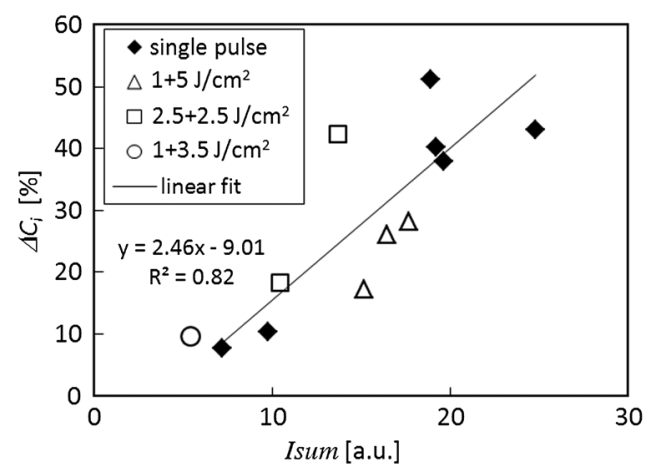

Fig. 8 Relation between the plasma radiation and the long term tattoo bleaching. In vivo experiment.

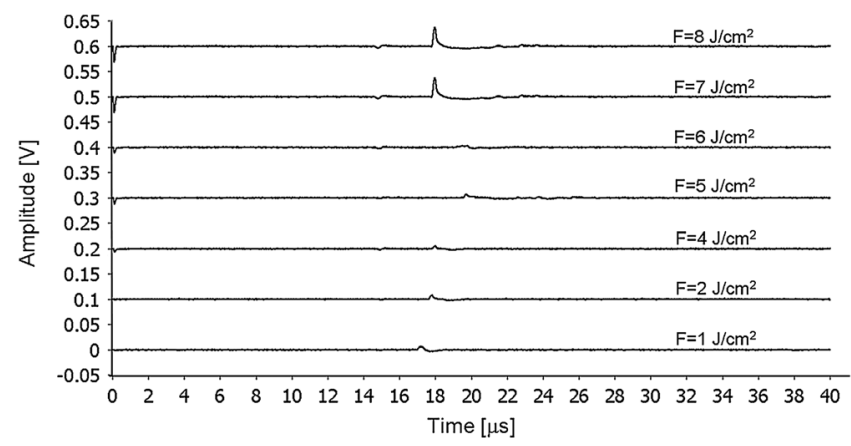

Fig. 9 The LBDP response of untattooed pig skin to laser pulses with different fluences.

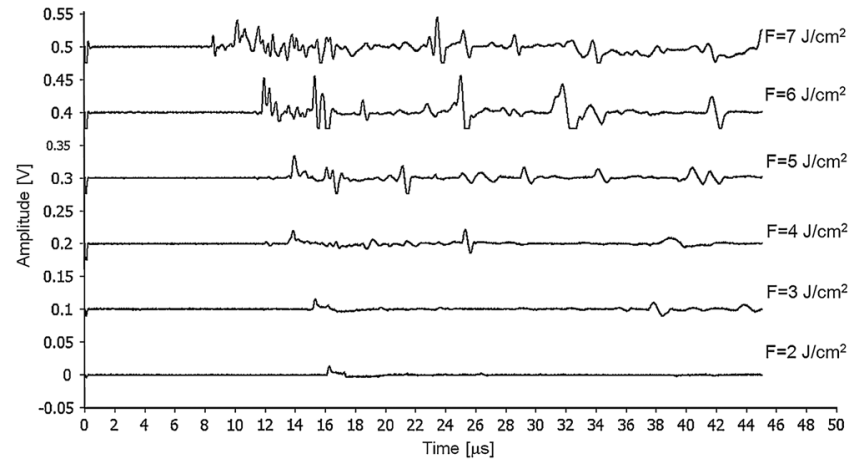

Fig. 10 The LBDP response of tattooed pig skin to laser pulses with different fluences.

with the signal from the untattooed pig skin. As in in vivo experiments, whitening was observed immediately after the laserpulse illumination in all cases. Furthermore, the skin perforation was observed at fluences of $4 \mathrm{~J} / \mathrm{cm}^{2}$ and above. This effect can also be seen in LBDP signals, where ejected particles cause signal oscillations following the shock wave. Compared with the in vivo experiments, the damage threshold for pig skin is at least $33 \%$ lower (4 versus $6 \mathrm{~J} / \mathrm{cm}^{2}$ ). We assume this decrease is caused by different tattoo properties and by the condition of the sample tissue, particularly by the loss of elasticity due to the heat treatment of the skin as a part of the slaughtering process. Similar damage might happen also in vivo on human skin if excessive fluence is used and the LBDP signal can be used to detect such severe damage when it does occur.

Results on the in vitro samples are similar to the results ex vivo. The samples were illuminated with approximately $30 \%$

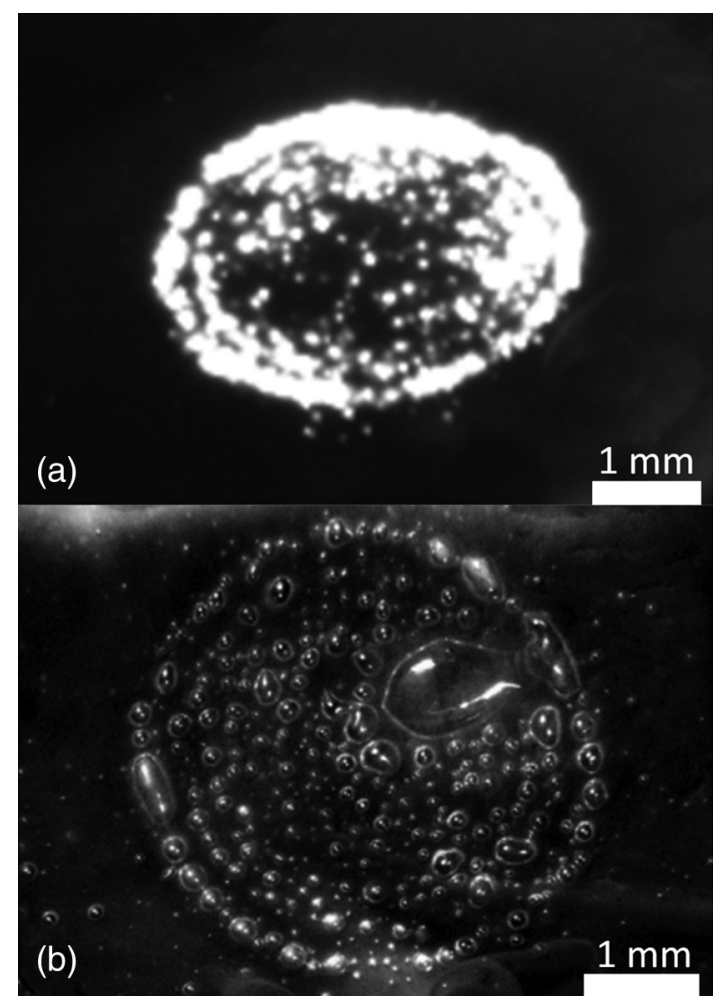

Fig. 11 Typical images of (a) plasma distribution and (b) bubbles, appearing in the in vitro samples. Laser beam diameter was $4 \mathrm{~mm}$ and the fluence $8 \mathrm{~J} / \mathrm{cm}^{2}$. 
higher fluences to obtain similar amplitudes of the LBDP signals. Due to the transparent collagen layer, some of the plasma effects were more clearly visible. We observed some hot spots in NIR images, which are mainly caused by small differences in irradiation and the absence of scatter [Fig. 11(a)]. At higher fluences $\left(>8 \mathrm{~J} / \mathrm{cm}^{2}\right)$ structural changes in the samples were observed. These could be seen as bubbles, located above the pigment layer, which did not disappear after the interaction [Fig. 11(b)]. We assume they contain gaseous decomposition products of the pigment. The same vacuolation was observed by Ferguson et al. ${ }^{9}$ on ex vivo samples. This could explain the increased scattering and the lower efficiency of the second laser pulse on the same spot. At even higher fluences, as measured ex vivo, the skin perforation could be accounted for by the gasses escaping from the skin surface.

From the experimental results it can be seen that the characteristic time, related to the cavitation bubbles and shock-waves dynamics, is in the range of $\mu \mathrm{s}$. The accompanying plasma lasts even less-approximately the same order as laser pulse time $\sim 100 \mathrm{~ns}^{21}$ On the other hand, the gaseous decomposition products in vacuolation structure, accompanied by a whitening of the impact area, last about $30 \mathrm{~min}^{23,24}$ The lymphatic elimination of fragmented pigment particles starts approximately 14 days after the treatment. ${ }^{11}$ In view of the listed time frames, it is evident that appropriate experimental methods were used.

\section{Conclusions}

Two methods of real-time monitoring of optodynamic phenomena that arise during laser tattoo removal are presented. The optodynamic waves, measured by the laser-beam deflection probe, include the information about the energy released during the interaction and is specific for different skin and tattoo conditions. The tissue ablation threshold for the untattooed and tattooed skin and the threshold for pigment removal can be determined. The optodynamic signals also show structural changes in the skin, which are otherwise difficult to detect. Skin perforation can be detected by the change of the signal form and the delayed signal due to particle ejection. The NIR camera provides valuable information on the intensity and the spatial distribution of the tattoo removal process by detecting the accompanying plasma. This improves the understanding of the process, particularly regarding hot spots and effects near the edges of the tattooed areas.

We estimate that these experiments are fundamental for the further development of the monitoring system. Both methods, the beam-deflection probe and the NIR camera, are appropriate for implementation in a closed-loop control system for the laser tattoo removal procedure.

Using the two monitoring sensors, we also studied the effects of double pulses. The results show that the strategy of using a weak prepulse, and the main pulse is less effective than using a single pulse. We assume that even a weak prepulse produces structural changes in the skin and reduces the efficiency of the main pulse. On the other hand, a strong correlation of the time-integrated plasma radiation and the long-term tattoo removal rate was also found, which means that each laser pulse that generates plasma also removes some tattoo pigment, being the first or the following one. However, further experimental studies are needed to prove these findings.

Comparing different types of samples we found that ex vivo pig skin and in vitro collagen specimens are a good replacement when the function response of new methods is to be measured. They can be used for testing ablation and perforation threshold detection. But in the case of long-term removal effects, only in vivo samples are appropriate.

\section{References}

1. R. R. Anderson and J. A. Parrish, "Selective photothermolysis-precise microsurgery by selective absorption of pulsed radiation," Science 220(4596), 524-527 (1983).

2. S. Choudhary et al., "Lasers for tattoo removal: a review," Laser. Med. Sci. 25(5), 619-627 (2010).

3. M. A. Adatto, "Laser tattoo removal: benefits and caveats," Med. Laser Appl. 19(4), 175-185 (2004).

4. E. F. Bernstein, "Laser treatment of tattoos," Clin. Dermatol. 24(1), 43-55 (2006).

5. T. C. Beute et al., "In vitro spectral analysis of tattoo pigments," Dermatol. Surg. 34(4), 508-516 (2008) .

6. D. D. Ho et al., "Laser-tattoo removal-a study of the mechanism and the optimal treatment strategy via computer simulations," Laser. Surg. Med. 30(5), 389-397 (2002).

7. E. V. Ross et al., "Comparison of responses of tattoos to picosecond and nanosecond $Q$-switched neodymium: YAG lasers," Arch. Dermatol. 134, 167-171 (1998).

8. L. Izikson et al., "Safety and effectiveness of black tattoo clearance in a pig model after a single treatment with a novel 758-nm 500 picosecond laser: a pilot study," Laser. Surg. Med. 42(7), 640-646 (2010).

9. J. E. Ferguson et al., "The $Q$-switched neodymium:YAG laser and tattoos: a microscopic analysis of laser-tattoo interactions," Br. J. Dermatol. 137(3), 405-410 (1997).

10. K. O'goshi, C. Suihko, and J. Serup, “In vivo imaging of intradermal tattoos by confocal scanning laser microscopy," Skin Res. Tech. 12(2), 94-98 (2006)

11. S. Irkoren et al., "The $Q$-switched Nd:YAG laser in tattoo removal and the effect of lymphatic elimination: an experimental study in rabbits," Surg. Sci. 2(5), 262-268 (2011).

12. C. R. Taylor et al., "Light and electron microscopic analysis of tattoos treated by $Q$-switched ruby laser," J. Invest. Dermatol. 97(1), 131-136 (1991).

13. H. Morsy et al., "Imaging of intradermal tattoos by optical coherence tomography," Skin Res. Tech. 13, 444-448 (2007).

14. M. Milanič and B. Majaron, "Spectral filtering in pulsed photothermal temperature profiling of collagen tissue phantoms," J. Biomed. Opt. 14(6) 064024-1-064024-8 (2009).

15. K. Nahen and A. Vogel, "Plume dynamics and shielding by the ablation plume during Er:YAG laser ablation," J. Biomed. Opt. 7(2), 165-178 (2002).

16. J. Diaci, "Response functions of the laser-beam deflection probe for detection of spherical acoustic-waves," Rev. Sci. Instrum. 63(11), 5306-5310 (1992).

17. B. Sullivan and A. C. Tam, "Profile of laser-produced acoustic pulse in a liquid," J. Acoust. Soc. Am. 75(2), 437-441 (1984).

18. J. T. Walsh and T. F. Deutsch, "Measurement of Er:YAG laser ablation plume dynamics," Appl. Phys. B 52(3), 217-224 (1991).

19. J. Možina and R. Hrovatin, "Optodynamics-a synthesis of optoacoustics and laser processing," Prog. Nat. Sci. 6, S709-S714 (1996).

20. B. Cencic et al., "High fluence, high beam quality Q-switched Nd: YAG laser with optoflex delivery system for treating benign pigmented lesions and tattoos," J. Laser Health Academy, 2010(1), 9-18 (2010).

21. A. Vogel and V. Venugopalan, "Mechanisms of pulsed laser ablation of biological tissues," Chem. Rev. 103(2), 577-644 (2003).

22. M. Jezeršek and J. Možina, "A laser anamorph profilometer," J. Mech. Eng. 49(2), 76-89 (2003).

23. L. Goldman et al., "Radiation from a $Q$-switched ruby laser. Effect of repeated impacts of power output of 10 megawatts on a tattoo of man," J. Invest. Dermatol. 44, 69-71 (1965).

24. T. Kossida et al., "Optimal tattoo removal in a single laser session based on the method of repeated exposures," J. Am. Acad. Dermatol. 66(2), 271-277 (2012). 